\title{
Cutaneous Pseudolymphoma Secondary to Exogenous Pigment in a Polychrome Tattoo
}

Mendez Diaz $\mathrm{Y}^{1}$, García-Arpa $\mathrm{M}^{2}$, Parra Cubillos $\mathrm{A}^{2}$, De Lara Simón $\mathrm{IM}^{3}$, García Rodriguez $\mathrm{R}^{1}$, Gómez Torrijos $\mathrm{E}^{1}$

${ }^{1}$ Allergology Section, Hospital General Universitario de Ciudad Real, Spain

${ }^{2}$ Dermatology Service, Hospital General Universitario de Ciudad Real, Spain

${ }^{3}$ Histopathology Service, Hospital General Universitario de Ciudad Real, Spain

J Investig Allergol Clin Immunol 2018; Vol. 28(3): 199-201 doi: $10.18176 /$ jiaci.0238

Key words: Cutaneous pseudolymphoma. Tattoo. Lymphocytes. Contact dermatitis.

Palabras clave: Pseudolinfoma cutáneo. Tatuaje. Linfocitos. Dermatitis de contacto.

Cutaneous lymphoid hyperplasia, or cutaneous pseudolymphoma (CPL), is a benign lymphoproliferative disorder of reactive T or B lymphocytes that clinically and/or histologically mimics cutaneous lymphoma [1]. The most frequent complications related to tattoos include infections, allergic and irritant contact dermatitis, and granulomatous dermatitis, mainly of the foreign body and sarcoid types. Pseudolymphomatous reactions are rarer, although their frequency may be underestimated [2]. Red pigment is the most common cause of reactions and pseudolymphomas due to tattoos [3-5].

We report the case of a patient with CPL associated with pink pigment in specific areas of a tattoo.

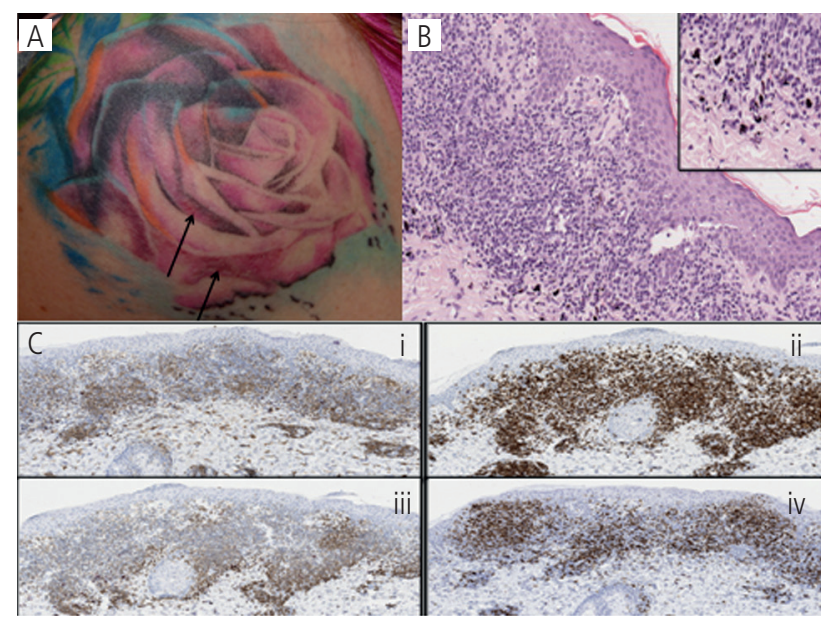

Figure. Papulovesicular lesions affecting only some pink areas of the tattoo (arrows). B, Skin tissue with a lymphohistiocytic infiltrate in the superficial dermis associated with an exogenous black-pink pigment deposit (hematoxylin-eosin, $\times 20$ ); the inset shows the pigment deposit in detail (hematoxylin-eosin, $\times 40$ ). C, Lymphocytes of the inflammatory infiltrate labeled using the immunohistochemical technique: i, CD4; ii, CD7; iii, CD2; iv, CD8 
A 25-year-old woman reported the appearance of pruritic lesions on her right shoulder at the site where a multicolored tattoo had been placed 2 months earlier. The lesions had been present for 2 weeks before she came to our clinic. The patient had had other black tattoos for 10 years, with no local reaction. Examination revealed a tattoo with green, black, and blue ink, as well as several shades of pink, with grouped papulovesicular lesions affecting only some pink areas of the tattoo (Figure).

No enlarged lymph nodes were palpated in the area of the tattoo. Biopsy of a papule revealed acanthosis with focal parakeratosis in the epidermis and a dense inflammatory infiltrate in the superficial dermis comprising lymphocytes (some with mild atypia), histiocytes, and some eosinophils. A disperse deposit of exogenous dark-pink pigment was observed in the interstitium. The deposit was phagocytosed by histiocytes (Figure). The immunohistochemical study showed that most of the lymphocytes were of the $\mathrm{T}\left(\mathrm{CD}^{+}\right)$strain, with predominant CD4 expression and a small accompanying population of B lymphocytes $\left(\mathrm{CD} 20^{+}\right)$. The lymphocytes maintained expression of CD2 and CD7 (Figure). These findings led us to diagnose T-lymphocyte CPL secondary to exogenous tattoo pigment. The patient underwent patch testing with the European standard and metal series and the pink pigment of the tattoo, which was provided by the patient. All the patch tests were negative except for nickel, for which the patient reported contact eczema induced by jewelry. The lesions disappeared spontaneously after 2 months.

During the last decade, there have been reports of allergic contact dermatitis to tattoos related to $p$-phenylenediamine [6]. In Spain, this substance, is the most commonly involved allergen in reactions affecting hairdressers (19.5\%), which is the occupation with the highest risk of reactions to $p$-phenylenediamine (26\%) [7].

The exact incidence of tattoo reactions is unknown. Tattooing has become very popular in both children and adults [8]. The tattoos are easily identifiable in the biopsy, since the pigment, which is usually distributed in the superficial and middle dermis, is seen in the form of extracellular deposits between the collagen bands and inside the macrophages. In addition to infections and neoplasms, tattoos can trigger nonspecific inflammatory reactions that affect the area of the tattoo or only a part of it. There may be different histological patterns, the lichenoid pattern being the most frequent [1]. The first case of CPL was reported in 1903, and, since then, approximately 40 cases have been reported. As in other inflammatory reactions to tattoos, the cutaneous manifestations are diverse and nonspecific, appearing mainly as macules, papules, or nodules $[2,4,5]$.

The lesions sometimes worsen with exposure to sunlight and excessive sweating. The latency period is variable (from 1 month to several years after, even as much as 30 years) [1-3]. The histological examination is characterized by a diffuse dermal infiltrate consisting mainly of macrophages and small lymphocytes (mainly T lymphocytes, although a predominance of $\mathrm{B}$ and mixed lymphocytes has also been reported). Eosinophils, plasma cells, histiocytes, and multinucleated giant cells may also be observed. The presence of macrophages phagocytizing the pigment is a key finding in the diagnosis, as is the polyclonal rearrangement of the lymphocytes [4].
While pathogenesis is unknown, antigenic stimulation by the exogenous pigment is thought to be responsible for sensitization $[2,4]$ and the delayed hypersensitivity reaction, which induces polyclonal proliferation of lymphocytes [2]. Red pigment is the most frequently implicated, although the reaction has also been described with green and blue pigments [1].

Complications of tattoos seem to be less frequent owing to improved techniques, more hygienic conditions, and less frequent use of sensitizing substances, such as mercury sulfide (red pigments), cadmium (yellow pigments), and cobalt (blue pigments), which are being replaced by more modern organic dyes $[1,5]$. In the case we report, we were unable to identify the components of the pink ink, although we believe that one of its components could be the culprit. Kluger et al found several metallic salts in the composition of the red ink, including zinc, copper, and nickel. In the present case, patch test results to nickel were positive, but we do not know if nickel was responsible for the CPL. Some tattoo inks analyzed contained nickel, although its causal relationship in individuals sensitized to this metal is not clear. In a series of 90 cases with inflammatory reactions to tattoos that were patch tested with standard, textile, and dye series, as well as with tattoo inks, positive results were observed in $29 \%$ of cases with the standard series (mainly to nickel), while results were positive for only $7 \%$ of the textile dyes and $11 \%$ of inks provided by the patient, thus indicating the limited role of contact tests in the diagnosis of tattoo reactions [9].

In the diagnosis of CPL caused by tattoos, the clinical and pathological correlation is fundamental, and the architecture and composition of the infiltrate, the presence of pigment, the immunohistochemistry results, and the absence of clonality have to be taken into account to enable a differential diagnosis with lymphoma [4].

There is no standard treatment for CLP induced by tattoo ink [3]. Topical or intralesional application of corticosteroids shows variable results $[2,3,5]$, with recurrences that can be explained by the persistence of the pigment in the dermis. Other lines of treatment include surgical removal and/or $\mathrm{CO}_{2}$ and YAG laser therapy [5]. Spontaneous remission has also been reported, as occurred in the present case. Progression towards malignancy is exceptional; to date, only 1 case has been reported, with a tattoo that contained mercury, probably as a result of chronic antigenic stimulation of B lymphocytes. Therefore, careful follow-up is recommended $[1,10]$.

We report a case of CPL as rare complication of a tattoo.

\section{Funding}

The authors declare that no funding was received for the present study.

\section{Conflicts of Interest}

The authors declare that they have no conflicts of interest.

\section{References}

1. Toro Montecinos M, Fernández-Figueras MT, Rivera Ruiz N. Patrones histológicos de reacciones cutáneas a tatuajes. Piel. 2015;30:443-8. 
2. Campolmi P, Bassi A, Bonan P, Cannarozzo G, Gola M, Rossi Degl'Innocenti D, et al. Cutaneous pseudolymphoma localized to black tattoo. J Am Acad Dermatol. 2011;65:55-7.

3. Marchesi A, Parodi PC, Brioschi M, Marchesi M, Bruni B, Cangi MG, et al. Tattoo ink-related cutaneous pseudolymphoma: a rare but significant complication. Case report and review of the literature. Aesthetic Plast Surg. 2014;38:471-8.

4. Romero-Pérez $D$, Blanes Martínez $M$, Encabo-Durán B. Pseudolinfomas cutáneos. Actas Dermosifiliogr. 2016;107:640-51.

5. Martins Cruz F, Lage D, Marega Frigerio R, Colombini Zaniboni M, Fávaro Arruda L. Reactions to the different pigments in tattoos: a report of two cases: An Bras Dermatol. 2010;85:708-11.

6. Gonzalo-Garijo MA, Fernández-Durán DA, Pérez-Calderón $R$, Sánchez-Carvajal J. Allergic contact dermatitis due to a temporary henna tattoo, a hair dye, and a marker pen. J Investig Allergol Clin Immunol. 2008;18(3):226-7.

7. Holguín-Gómez L, Sastre Domínguez J.Occupational Contact Dermatitis in Spain. I Investig Allergol Clin Immunol. 2017;27(2):134-6.

8. Pegas JR, Criado PR, Criado RF, Vasconcellos C, Pires MC. Allergic contact dermatitis to temporary tattoo by p-phenylenediamine. J Investig Allergol Clin Immunol. 2002;12(1):62-4.

9. Serup J, Hutton Carlsen K. Patch test study of 90 patients with tattoo reactions: negative outcome of allergy patch test to baseline batteries and culprit inks suggests allergen(s) are generated in the skin through haptenization. Contact Dermatitis. 2014;71:255-63.

10. Gutermuth J, Hein R, Fend F, Ring J, Jakob T. Cutaneous pseudolymphoma arising after tattoo placement. J Eur Acad Dermatol Venereol. 2007;21:566-7.

I Manuscript received December 4, 2017; accepted for publication February 12, 2018.

Elisa Gomez Torrijos

Hospital General Universitario de Ciudad Real

C/ Obispo Rafael Torija, s/n

13005 Ciudad Real, Spain

E-mail: egomezt.cr@gmail.com 